\title{
A new technique in laparoscopic abdominal access (Evsen Method, Modified Veress Technique)
}

\author{
Mehmet Siddik Evsen, Mehmet Sait Icen, Fatih Mehmet Findik, Senem Yaman Tunc, \\ Elif Ağaçayak, Talip Gul
}

Dicle University, Diyarbakir/Turkey

\begin{abstract}
Objectives: The most important step in laparoscopic surgery is to safely establish the pneumoperitoneum, especially since approximately half of the complications occur during the initial entry into the abdomen. There is a distinct need to modify the available methods to reduce therate of adverse events in laparoscopic entry. In this study, a modified Veress technique (MVT) or Evsen method is introduced.

The aim of this article was to present a modified Veress technique for establishing the pneumoperitoneum.

Material and methods: The study was conducted at the Dicle University, Faculty of Medicine, Department of Obstetrics and Gynecology, from September 2016 to May 2017. A new laparoscopic entry technique was introduced and compared with the classical Veress technique. A total of 40 cases were included in the study. MVT and the classical Veress method were applied to 26 and 14 patients, respectively.

Results: The pneumoperitoneum was established at the first attempt in 23 (88.5\%) MVT patients and in 7 (50\%) patients from the classical Veress method group. The number of insufflation attempts to establish a successful pneumoperitoneum was lower using MVT and the difference was statistically significant (p: 0.022). As far as time is concerned, a comparison between the groups revealed that the pneumoperitoneum was established in a statistically significantly shorter time using MVT $(p<0.00)$.

Conclusions: The modified Veress technique proved to be superior to the classical Veress method for establishing the pneumoperitoneum. Using the new method, the pneumoperitoneum was established after fewer attempts and in a shorter time. Key words: laparoscopic entry, laparoscopic surgical technique, pneumoperitoneum, Veress needle
\end{abstract}

Ginekologia Polska 2018; 89, 9: 481-484

\section{INTRODUCTION}

Laparoscopic surgery has been increasingly used for the abdominal access. A safe establishment of the pneumoperitoneum remains the most important step in laparoscopic surgery. The risk of severe complications is very low in laparoscopic surgery and $50 \%$ of the complications occur during the initial entry into the abdomen. Large vessel injury and bowel injury during the first entry into the abdomen are the most feared complications and a source of anxiety for surgeons performing laparoscopy [1, 2].

Closed (Veress needle; direct trocar insertion) and open (Hasson) laparoscopic access techniques are the most com- mon methods used to establish the pneumoperitoneum [1, 3-5]. Each method has its own advantages, risks and disadvantages during the abdominal entry. Despite a significant number of studies in the literature, with large series, and comparisons of the available methods, there is no consensus which method should be used for the abdominal access [6, 7]. Therefore, there is a clear need for new methods, ideas and studies to modify the current methods to increase the safety of the procedures and reduce the rate of adverse events.

The purpose of the study was to present the modified Veress technique (MVT), which we used to establish the 
pneumoperitoneum. Moreover, the MVT and the classical Veress technique were compared.

\section{MATERIAL AND METHODS}

This study was conducted at the Dicle University, Faculty of Medicine, Department of Obstetrics and Gynecology, from September 2016 to May 2017. Local Ethics Committee approved of the study.

As far as practical training on laparoscopic abdominal access is concerned, instructors at our clinic train the assistants how to establish the pneumoperitoneum with the Veress technique, and place the trocar in the peritoneal cavity, during years 3 and 4 of their training. The assistants at the Gynecology and Infertility clinic received training on how to insert the Veress needle from a skin incision through subcutaneous, fascial and sub-peritoneal areas and peritoneum for insufflation of the peritoneal cavity. In this study, closed abdominal access with the Veress technique (Group I) and abdominal access with MVT (Group II) were compared. During the study period, 6 residents established the pneumoperitoneum with the Veress technique and MVT under the supervision of the instructors. The Veress needle was inserted into the abdomen at 90 degrees for MVT, while for the classical Veress entry the needle was inserted at 45 and 90 degrees in thin and overweight patients, respectively. The groups were compared for demographic data, number of attempts for establishing the pneumoperitoneum successfully, and time until a successful attempt. Time was calculated as time from the advancement of the Verres needle into the peritoneal cavity until confirmation of entry. Abdominal incision and detachment of the subcutaneous tissues were not included in the calculation of time. A successful pneumoperitoneum was described as follows: 1). no content coming from the tip of the Veress needle outside the abdomen when negative pressure is applied after it is progressed into the peritoneal cavity, 2). 3 cc saline

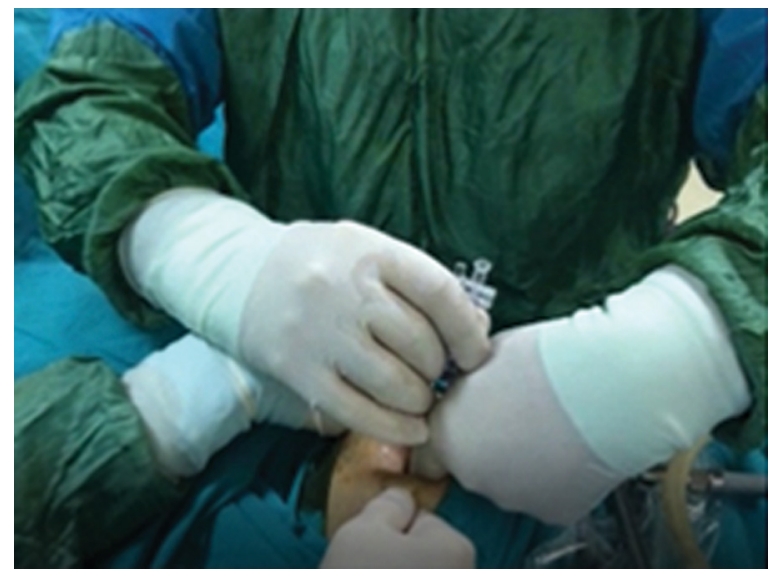

Figure 1. Umbilical region, Veress needle inserted with the guidance of the tip of the index finger solution injected passed smoothly and did not return during reaspiration, 3). peritoneal cavity pressure of $10 \mathrm{~mm} \mathrm{Hg}$, when measured with an insufflator. Homogenous distribution of gas after the establishment of the pneumoperitoneum for 2-3min, and absence of liver dullness were also considered to be additional determinants of a successful procedure [8]. In case of failure, the abovementioned procedure was repeated. The success of the procedure or the number of failed insufflation attempts until the procedure was performed successfully were recorded. After three failed insufflation attempts, the pneumoperitoneum was established by the instructors for the failed cases. During the study, the residents were not informed that MVT was a new method.

\section{Surgical technique}

In both methods, on the midline abdomen, the anterior abdominal wall was lifted up with the Kocher clamp at a depth of around $1-3 \mathrm{~cm}$ from the left and right lateral sides at a distance of $3 \mathrm{~cm}$ to the skin incision. In classical Veress technique, a $1 \mathrm{~cm}$ incision was made in the midline subumbilical or supraumbilical region. The Veress needle was advanced through the subcutaneous part and the anterior wall of the abdomen and the abdomen was insufflated.

\section{Modified Veress Technique (Evsen Method)}

For the abdominal access, a subumbilical or a supraumbilical skin incision was made with a scalpel at a distance of $1-1.3 \mathrm{~cm}$. Until the anterior abdominal wall fascia is felt with the tip of the index finger of the left hand (or inactive hand), subcutaneous tissue and subcutaneous fascia were dissected bluntly or sharply with scissors. After the fascia of the anterior abdominal wall was felt with the tip of the index finger, the Veress needle was inserted into the fascia of the anterior abdominal wall at an angle of 90 degrees under the guidance of the fingertip (Fig. 1, 2). The peritoneal

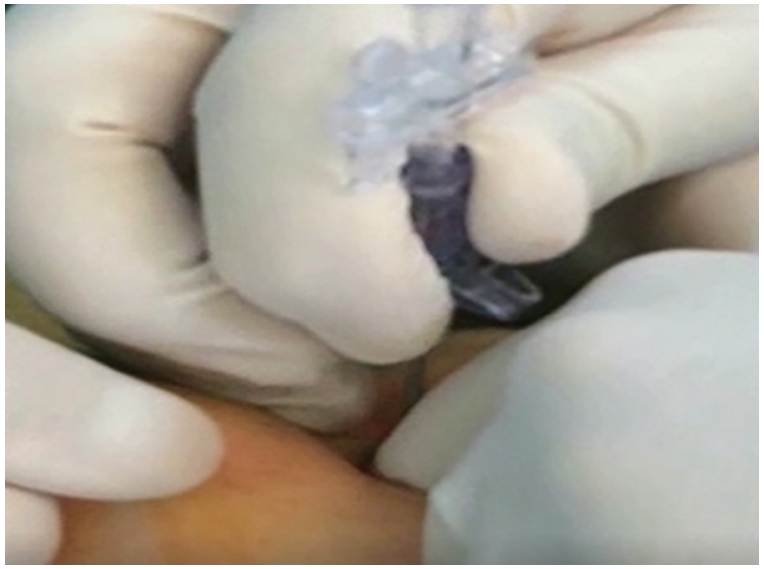

Figure 2. The tip of the index finger is touching the anterior abdominal wall fascia 
cavity was entered after the Veress needle was advanced $2-3 \mathrm{~cm}$ under the guidance of the tip of the index finger. The method can be followed on YouTube using the phrase: Evsen method [9]. Briefly, this new method could be briefly explained as follows: Modified Veress technique or Veress technique with the guidance and control of the index finger.

\section{Statistical analysis}

Statistical evaluation of data was done using IBM SPSS 21.0 for the Windows statistical package program. For the statistical assessment of data, the measurable variables were presented as mean \pm standard deviation (SD), while the categorical variables were presented as numbers and percentage (\%). We checked if the data followed a normal distribution. Student t-test was used for inter-group comparison of quantitative variables that had normal distribution. Measurement variables were presented -group comparison of qualitative data, Chi-square ( $\mathrm{x} 2$ ) test was used. Two-way hypotheses were used. The $p$-value of $<0.05$ was considered as statistically significant.

\section{RESULTS}

MVT and the classical Veress method to establish the pneumoperitoneum were applied to 26 and 14 patients, respectively. No differences in age and body mass index were found between the groups (Tab. 1).

The pneumoperitoneum was established at the first attempt in 23 (88.5\%) patients in Group II and 7 (50\%) patients in Group I. For a successful pneumoperitoneum, the number of insufflation attempts was lower in Group II and the difference was statistically significant (Tab. 2).

Mean and standard deviation for time needed until the successful attempt was $13.92 \pm 2.67$ seconds in Group II and $20.3 \pm 4.7$ seconds in Group I. As far as time is concerned, a comparison between the groups revealed that the pneu-

\begin{tabular}{|l|l|l|l|}
\hline \multicolumn{4}{|c|}{ Table 1. Comparison of demographic data between the groups } \\
\hline & Group I (n: 14) & Group II (n: 26) & p \\
\hline $\begin{array}{l}\text { Age [years] } \\
\text { (mean } \pm \text { SD) }\end{array}$ & $37.6(9.8)$ & $40.2(14.5)$ & 0.55 \\
\hline Body mass index & $28.1(4.8)$ & $30.3(6.3)$ & 0.26 \\
\hline
\end{tabular}

SD — Standard Deviation; $\mathrm{n}$ - number of patients

\begin{tabular}{|c|c|c|c|}
\hline & Group I (n: 14) & Group II (n: 26) & p \\
\hline Attempt (first) & 7 & 23 & \multirow{3}{*}{0.022} \\
\hline Attempt (second) & 3 & 2 & \\
\hline Attempt (third) & 4 & 1 & \\
\hline
\end{tabular}

moperitoneum was established in a statistically significantly shorter time in the MVT group $(p<0.00)$.

\section{DISCUSSION}

While selecting an abdominal access technique in laparoscopic surgery, surgeons will obviously prefer a technique with a lower complication rate, so the surgery can be performed in a safer and faster way. Surgeons will also select a technique based on their training, and with which they can feel more comfortable. The choice will be easier after comparing the available methods with larger series of patients. Despite various reports in the literature, there is no consensus which abdominal access method (open, closed access and access with optical trocar) should be recommended and there is a clear need for a new method in laparoscopic surgery [6].

In endoscopic surgery, gynecologic surgeons often prefer the method of establishing the pneumoperitoneum with the Veress needle $[10,11]$. In the Veress method, there is a certain degree of risk of failure in establishing the pneumoperitoneum, as well as the risk of subcutaneous air leakage. We believe that the new method we present in this study has some advantages over other methods. With MVT, the skin and the fascia are detached using sharp/blunt dissection, while the subcutaneous fascia (scarpa) is also passed through. Therefore, the area between the subcutaneous part and the fascia can be insufflated in the classical method, whereas this risk is minimal in the MVT group. In the classical Veress method (especially for new performers), the subcutaneous anterior abdominal wall emphysema may develop since gas may be injected before the needle is advanced adequately. We believe that the risk will be very low in case of MVT. However, there is a need for further studies on this matter. In MVT, the insertion angle of the Veress needle was 90 degrees, which in our opinion contributed to the decreased number of failed insufflation attempts.

Our study was not without limitations, chief among them a small sample size, although it was enough for statistical significance. Regardless, this study presents a new method in laparoscopic surgery. Obviously, there is a clear need for further studies, with larger series, to learn and popularize this method. The rate of minor and major complications has been reported to be low in laparoscopic surgery [1]. During the study period, we observed that the new method was more comfortable and successful as compared to the classical Veress method. We considered it would be good medical practice to not increase the number of patients in the control group. Additionally, while groups were compared, the new method was found to be statistically more advantageous so, the number of controls was terminated in the classical Veress group. Furthermore, we think that the low number of complications in this study was associated with the low number of patients. 
After establishing the pneumoperitoneum with gas, the performer could touch the anterior abdominal wall with the index finger. Since the skin incision was a little longer in the new method as compared to the classical method (incision needed to be long enough for the entry of the left index finger and insertion of the Veress needle), it may be possible to determine how far the trocar can be advanced by evaluating the thickness of the anterior wall of the abdomen. We observed that the skin was passed more smoothly during the initial trocar placement and this will probably decrease the likelihood of increased uncontrolled initial trocar placement, which indeed could be an advantage.

Although a large incision might slightly increase the risk of gas leakage and displacement of the trocar during the surgical procedure, this disadvantage can be overcome by a simple suture to fix the incision and the trocar.

It is important for the surgeons performing the procedure to feel more comfortable and advance the Veress needle under better control. A better-controlled entry will decrease anxiety and the surgeons will feel more at ease. Our study was conducted with the surgeons who only recently learned the technique and improved their confidence. We think that the technique, once it is mastered and surgeons gain sufficient experience, will become the preferred method.

The open (Hasson) method is not preferred since the time to enter the peritoneal cavity and the learning curve are longer, the rate of gas leakage is higher, and surgeons performing gynecological endoscopy have limited experience with the method $[5,10,11]$. With direct trocar insertion, the pneumoperitoneum can be established faster as compared to the open and classical Veress method. However, the required experience and the likelihood of causing a possible injury, with a large defect, may be the source of much anxiety among the surgeons. We found that the pneumoperitoneum could be established faster using MVT as compared to the classical Veress technique. Moreover, it is associated with a lower number of failed insufflation attempts. We think that MVT will be preferred instead of the classical Veress technique because of the above mentioned advantages. Also, it is faster and has a shorter learning curve as compared to the other methods. We guess that MVT will cause less anxiety compared to the direct trocar insertion.

\section{CONCLUSIONS}

We suppose that MVT (Evsen method) will offer an interesting alternative for practitioners, especially those who have trouble entering the abdomen. The choice of a method will be clearer after a large series comparison of the available methods. We think that MVT is more advantageous than the other methods for establishing the pneumoperitoneum. More accurate data can be obtained from further studies, once the method is internalized and used more commonly.

\section{REFERENCES}

1. Krishnakumar S, Tambe P. Entry complications in laparoscopic surgery. J Gynecol Endosc Surg. 2009; 1(1): 4-11, doi: 10.4103/0974-1216.51902, indexed in Pubmed: 22442503.

2. Philips PA, Amaral JF. Abdominal access complications in laparoscopic surgery. J Am Coll Surg. 2001; 192(4): 525-536, indexed in Pubmed: 11294410.

3. Toro A, Mannino M, Cappello G, et al. Comparison of two entry methods for laparoscopic port entry: technical point of view. Diagn Ther Endosc. 2012; 2012: 305428, doi: 10.1155/2012/305428, indexed in Pubmed: 22761542.

4. Y-Palmer R. Safety in laparoscopy. J Reprod Med. 1974; 13: 1-5.

5. Hasson HM, Rotman C, Rana N, et al. Open laparoscopy: 29-year experience. Obstet Gynecol. 2000; 96(5 Pt 1): 763-766, indexed in Pubmed: 11042315.

6. Ahmad G, Gent D, Henderson D, et al. Laparoscopic entry techniques. Cochrane Database of Systematic Reviews. 2015, doi: 10.1002/14651858.cd006583.pub4.

7. Cornette B, Berrevoet F. Trocar Injuries in Laparoscopy: Techniques, Tools, and Means for Prevention. A Systematic Review of the Literature. World J Surg. 2016; 40(10): 2331-2341, doi: 10.1007/s00268-016-3527-9, indexed in Pubmed: 27146054.

8. Teoh B, Sen R, Abbott J. An evaluation of four tests used to ascertain Veres needle placement at closed laparoscopy. J Minim Invasive Gynecol. 2005; 12(2): 153-158, doi: 10.1016/j.jmig.2005.01.011, indexed in Pubmed: 15904620.

9. https://www.youtube.com/watch? $\mathrm{v}=\mathrm{F} 5 \mathrm{~S} 64 \mathrm{U} 5 \mathrm{vIM}$

10. Kroft J, Aneja A, Tyrwhitt J, et al. Laparoscopic peritoneal entry preferences among Canadian gynaecologists. J Obstet Gynaecol Can. 2009; 31(7): 641-648, indexed in Pubmed: 19761637.

11. Lalchandani S, Phillips K. Laparoscopic entry technique-a survey of practices of consultant gynaecologists. Gynecological Surgery. 2005; 2(4): 245-249, doi: 10.1007/s10397-005-0143-3. 